\title{
Novel classes of integers and their applications in graph labeling
}

\author{
Shahbaz Ali*1,2 (D), Muhammad Khalid Mahmood ${ }^{1}$ (D), Kar Ping Shum ${ }^{3}$ (D) \\ ${ }^{1}$ Department of Mathematics, University of the Punjab, Lahore 54590, Pakistan \\ ${ }^{2}$ Department of Mathematics, Khwaja Fareed University of Engineering and Information Technology, \\ Rahim Yar Khan 64200, Pakistan \\ ${ }^{3}$ School of Mathematics and Statistics, Southwest University Chongqing, Beibai, P.R.China
}

\begin{abstract}
Adding new classes of integers to literature is both challenging and charming. Until a new class is completely characterized, mathematics is never going to be worth it. While it's absurd to play with integers without intended consequences. In this work, we introduce and investigate four new classes of integers namely, anti-totient numbers, half anti-totient numbers, near Zumkeller numbers and half near Zumkeller numbers by using the notion of non-coprime residues of $n$ including $n$. We formulate and propose relations of these new classes of numbers with previous well-known numbers such as perfect, totient, triangular, pentagonal, and hexagonal numbers. These new classes of integers have been completely characterized. Finally, as an application of these new classes of numbers, a new graph labeling is also proposed on anti-totient numbers.
\end{abstract}

\section{Mathematics Subject Classification (2020). 11A25, 05C78}

Keywords. anti-totient number, half anti-totient number, near Zumkeller number, half near Zumkeller number, graph labeling

\section{Introduction}

A positive integer $n$ is termed as a perfect number if the sum of its positive proper divisors is $n$. That is, $\sigma(n)=n$, where $\sigma(n)$ represents the sum of all positive proper divisors of $n$. The generalized perfect numbers are called Zumkeller numbers. Zumkeller numbers generated a sequence of integers in which the positive divisors of every integer can be partitioned into two sets whose sums are equal [4]. The notion of Zumkeller numbers was formally investigated by Clark et al. in [4]. Later on, Peng and Bhaskara Rao proved several postulates about Zumkeller numbers in [13]. The notion of totient, super totient and hyper totient numbers was introduced by Khalid and Shahbaz in [15]. An integer $n$ greater than one is known as totient if the sum of its co-prime residues is $2^{k} n, k \geq 1$. Further, we refer it as super totient if the set of its co-prime residues can be partitioned into two disjoint subsets of equal sums. Moreover, if the set its of co-prime residues including $n$ itself can also be divided into two disjoint subsets of equal sums, then it is known as hyper

\footnotetext{
*Corresponding Author.

Email addresses: shahbaz.ali@kfueit.edu.pk (S. Ali), khalid.math@pu.edu.pk (M.K. Mahmood), kpshum@ynu.edu.cn (K.P. Shum)

Received: 13.11.2020; Accepted: 24.02.2021
} 
totient. In [15], it was shown that every Zumkeller number is either a super totient or hyper totient number. Many postulates and relations on totient, super totient and hyper totient numbers were developed in [15].

The above newly defined classes can also be used in graph labeling. Labeled graphs have many applications in X-ray crystallography, coding theory, circuit design communication networks, etc. In [14], the concept of graph labeling was investigated by Rosa in 1967 for the very first time. Eshghi, Kourosh and Parham presented applications of mathematical programming using graph labeling in [6]. Babitha and Baskar proved some results on prime cordial labeling in [1]. The mean graph labeling was introduced by Somasundaram and Ponraj in [18]. Balamurugan, Thirusangu and Thomas introduced the notion of Zumkeller labeling in [2]. Hussain and Tabraiz investigated Super d-antimagic labeling of subdivided $k C_{5}$ in [10]. In [16], some results and examples on different cordial graphs were determined by Seoud and Salman. In [5], Dinh and Moshe investigated a new labeling of $C_{2 n}$. More results on edge-odd graceful graphs was given by Seoud and Maher in [17]. In [11,12], Khalid and Shahbaz introduced the concept of super totient labeling on various classes of graphs. In [9], Harrington, and Tony investigated restricted super totient labeling and they found their indices as well. A detailed survey on graph labelings has been explored by Gallian in [7].

This paper is arranged as follows: In Sections 2 and 3, we introduce the notion of antitotient and half anti-totient numbers respectively. Also, we give many results on their relations with existing classes of integers. In Section 4, we define near Zumkeller and half near Zumkeller numbers by means of two disjoint subsets of equal sum over non-coprime residues of any positive integer $n$. In Section 5, we give an application of anti-totient numbers in graph labeling and also propose an algorithm for anti-totient labeling of a full $\mathrm{K}$-ary tree. Some results are given below to use in the sequel.

Definition 1.1. [3] Let $r$ be the residue of any postive integer $n$. We say that $r$ and $n$ are co-prime to each other if $(r, n)=1$ (where $(r, n)$ represents the greatest common divisor (GCD) of $r$ and $n$ ). The Euler's phi function of a positive integer $n$ counts the number of co-prime residues of $n$, it is denoted by $\varphi(n)$.

Proposition 1.2. [15] An integer $n>0$ is totient if and only if $\varphi(n)=2^{k+1}, k \geq 1$.

Proposition 1.3. [13] If $n$ is a Zumkeller number, then

(a) $\sigma(n)$ is even.

(b) The prime factorization of $n$ must include at least one odd prime to an odd power.

Note: Throughout the paper $\mathbb{Z}, \mathbb{N}=\mathbb{Z}^{+}, \mathbb{E}^{+}, \mathbb{O}^{+}$represents the set of all integers, set of all positive integers, set of all even and odd positive integers respectively.

\section{Anti-totient numbers}

In this section, we define the notion of anti-totient numbers and characterize them completely. Also, we establish relations of anti-totient numbers with well-known classes of integers.

Definition 2.1. A positive integer $n$ is called an anti-totient number if the sum of its non co-prime residues including $n$ is

$$
\xi(n)=\sum_{\substack{(r, n) \neq 1, r \leq n}} r= \begin{cases}n k, & \text { if } n \text { is odd and for some even } k, \\ \frac{3 n}{2} k, & \text { if } n \text { is even and for some odd } k .\end{cases}
$$


Example 2.2. Let $n=26$ be an integer. The set of non co-prime residues of 26 including 26 is $S=\{2,4,6,8,10,12,13,14,16,18,20,22,24,26\}$. The sum of the elements of $S$ is $195=\frac{3 \cdot 26}{2} \cdot 5$. For $n=35$, the set of non co-prime residues of 35 including itself is $T=\{5,7,10,14,15,20,21,25,28,30,35\}$. The sum of elements of $T$ is $210=35 \cdot 6$. If we take $n=10$, then the sum of its co-prime residues including itself is 35 which can not be written in the form of $\frac{3 \cdot 10}{2} \cdot k$ for any odd integer $k$. Therefore, 26 and 35 are even and odd anti-totient numbers respectively, but 10 is not an anti-totient number.

To characterize the set of all anti-totient numbers, we give the following important lemma.

Lemma 2.3. An integer $n$ is an anti-totient number if and only if

$$
\varphi(n) \equiv \begin{cases}n+1(\bmod 4), & \text { if } n \text { is odd } \\ n-2(\bmod 6), & \text { if } n \text { is even. }\end{cases}
$$

Proof. Let $\left\{r_{1}, r_{2}, \cdots, r_{n-1}\right\}$ and $\left\{r_{1}^{\prime}, r_{2}^{\prime}, \cdots, r_{\varphi(n)}^{\prime}\right\}$ be sets of all residues and co-prime residues of $n$ respectively. The sum of all residues including $n$ and co-prime residues of $n$ are $n(n+1) / 2$ and $n \varphi(n) / 2$ respectively. Thus the sum of all non co-prime residues of $n$ is

$$
\xi(n)=\sum_{i=1}^{n-1} r_{i}-\sum_{i=1}^{\varphi(n)} r_{i}^{\prime}=\frac{n(n+1)}{2}-\frac{n \varphi(n)}{2}=\frac{n}{2}(n+1-\varphi(n)) .
$$

If $n$ is an odd, then $\frac{n}{2}(n+1-\varphi(n))=n k, \Rightarrow 2 k=(n+1-\varphi(n))$. So, $k$ is even if and only if $\varphi(n) \equiv n+1(\bmod 4)$.

Now, if $n$ is an even integer. Then,

$$
\xi(n)=\frac{n(n+1)}{2}-\frac{n \varphi(n)}{2}=\frac{n}{2}(n+1-\varphi(n))=3 n k / 2,
$$

$\Rightarrow n+1-\varphi(n)=3 k$. Therefore, an integer $k$ is odd if and only if $n-2 \equiv \varphi(n)(\bmod 6)$.

Theorem 2.4. Let $p$ be an odd prime and $\alpha$ be any positive integer. The integer $p^{\alpha}$ is an anti-totient number if and only if $\alpha$ is even and $p \equiv 3(\bmod 4)$.

Proof. The sum of non co-prime residues of $p^{\alpha}$ including $p^{\alpha}$, is $\xi\left(p^{\alpha}\right)=\frac{p^{\alpha}}{2}\left(p^{\alpha}+1-\varphi\left(p^{\alpha}\right)\right)$. Since $p^{\alpha}$ is an odd prime, we have that

$$
\begin{aligned}
\frac{p^{\alpha}}{2}\left(p^{\alpha}+1-\varphi\left(p^{\alpha}\right)\right) & =2 p^{\alpha} k, \\
\Rightarrow \quad 1+p^{\alpha-1} & =4 k .
\end{aligned}
$$

We need to show that $k$ is even in Equation $(2.1)$ if and only if $p \equiv 3(\bmod 4)$ and $0 \neq \alpha \equiv 0(\bmod 2)$. There are four possibilities with respect to $p$ and $\alpha$. These are

$$
\begin{aligned}
& (a) p \equiv 1(\bmod 4) \text { and } 0 \neq \alpha \equiv 0(\bmod 2), \\
& (b) p \equiv 3(\bmod 4) \text { and } \alpha \equiv 1(\bmod 2), \\
& (c) p \equiv 1(\bmod 4) \text { and } \alpha \equiv 1(\bmod 2), \\
& (d) p \equiv 3(\bmod 4) \text { and } 0 \neq \alpha \equiv 0(\bmod 2) .
\end{aligned}
$$

(a) : Setting $p=1+4 t$ for some positive integer $t$ and $\alpha=2 m, m \in \mathbb{Z}^{+}$in Equation (2.1),

$$
1+(1+4 t)^{2 m-1}=4 k .
$$

This is not possible because $1+(1+4 t)^{2 m-1}$ is not divisible by 4 . Similarly, possibilities (b) and $(c)$ do not hold. When $p=3+4 t$ then by Lemma $2.3, p^{\alpha}$ is an anti-totient number if and $\alpha$ is an even.

Theorem 2.5. Let $p_{i}, q_{i}$ be primes of the form $p_{i}=1+4 t_{i}$ and $q_{i}=3+4 t_{i}$. The integer $n=\prod_{i=1}^{m} p_{i}^{\alpha_{i}} q_{i}^{\beta_{i}}$ is an anti-totient number if and only if there is an odd number of $\beta_{i}$ 's with $\beta_{i} \equiv 1(\bmod 2)$ for each $i$. 
Proof. Let $n=\prod_{i=1}^{m} p_{i}^{\alpha_{i}} q_{i}^{\beta_{i}}$ be an odd integer with primes $p_{i}, q_{i}$ of the form $1+4 t_{i}$ and $3+4 t_{i}$ respectively for some positive integers $t_{i}$. Let's assume an integer,

$$
\begin{aligned}
\gamma & =\prod_{i=1}^{m} p_{i}^{\alpha_{i}} q_{i}^{\beta_{i}}+1-\varphi\left(\prod_{i=1}^{m} p_{i}^{\alpha_{i}} q_{i}^{\beta_{i}}\right) \\
& =\prod_{i=1}^{m} p_{i}^{\alpha_{i}} q_{i}^{\beta_{i}}+1-\prod_{i=1}^{m}\left(p_{i}^{\alpha_{i}-1}\left(p_{i}-1\right) q_{i}^{\beta_{i}-1}\left(q_{i}-1\right)\right) .
\end{aligned}
$$

Since $p_{i} \equiv 1(\bmod 4)$ and $q_{i} \equiv 3(\bmod 4)$, we have that

$$
\begin{aligned}
\gamma & \equiv \prod_{i=1}^{m} q_{i}^{\beta_{i}}+1(\bmod 4) \\
& \equiv \prod_{i=1}^{m} 3^{\beta_{i}}+1(\bmod 4)
\end{aligned}
$$

from Congruence (2.3), it is clear that the integer $\gamma$ is divisible by 4 if and only if $\beta_{i}$ are odd in numbering $(m \equiv 1(\bmod 2))$ with $\beta_{i} \equiv 1(\bmod 2)$.

Corollary 2.6. Let $p_{i}, q_{i}$ be primes of the form $p_{i}=1+4 t_{i}$ and $q_{i}=3+4 t_{i}$, then $n=\prod_{i=1}^{m} p_{i} q_{i}$ is an anti-totient number if and only if $m \equiv 1(\bmod 2)$.

Corollary 2.7. Let $q_{i}$ be primes of the form $q_{i}=3+4 t_{i}$, then $n=\prod_{i=1}^{m} q_{i}^{\beta_{i}}$ is an anti-totient number if and only if $1 \neq m \equiv 1(\bmod 2)$ and $\beta_{i} \equiv 1(\bmod 2)$.

Corollary 2.8. The product of two odd primes $p$ and $q$ is anti-totient if and only if $p \equiv 1(\bmod 4)$ and $q \equiv 3(\bmod 4)$.

Remark 2.9. Let $p_{i}$ be primes of the form $1+4 t_{i}$ for some positive integers $t_{i}$ then the product $\prod_{i=1}^{m} p_{i}^{\alpha_{i}}$ is not an anti-totient number.

Proposition 2.10. An integer $2^{\alpha}$ is an anti-totient number if and only if $0 \neq \alpha \equiv$ $0(\bmod 2)$.

Proof. Let $n=2^{\alpha}$ be an integer. Let us assume that an integer $\gamma$ as

$$
\begin{aligned}
\gamma & =2^{\alpha}-2-\varphi\left(2^{\alpha}\right)=2^{\alpha}-2-2^{\alpha-1} \\
& =2\left(2^{\alpha-1}-1-2^{\alpha-2}\right) .
\end{aligned}
$$

Since,

$$
2^{\alpha}(\bmod 6)= \begin{cases}2, & \text { if } \alpha \text { is odd }, \\ 4, & \text { if } \alpha \text { is even. }\end{cases}
$$

Therefore, the integer $\gamma$ is divisible by 6 if and only if $\alpha \equiv 0(\bmod 2)$.

Proposition 2.11. Let $p$ be an odd prime of the form $p=1+4 t$ or $p=3+4 t$. An integer $2^{\alpha} p$ is anti-totient if and only if $t \in\left\{3 r, 3 r+5 \mid r \in \mathbb{Z}^{+} \cup\{0\}\right\} \cup\left\{(3 z-1) / 2 \mid z \in \mathbb{Z}^{+}\right\}$.

Proof. Let $p$ be an odd prime of the form $\left.1+4 t, t \in \mathbb{Z}^{+}\right\}$. Take the integer

$$
\begin{aligned}
\gamma & =2^{\alpha} p-2-\varphi\left(2^{\alpha} p\right)=2^{\alpha} p-2-2^{\alpha-1} p+2^{\alpha-1} \\
& =2^{\alpha}+2^{\alpha+2} t-2-2^{\alpha+1} t .
\end{aligned}
$$

If $\alpha$ is even then Equation (2.5) becomes

$$
\gamma \equiv 2 t+2(\bmod 6)
$$


The integer $\gamma$ is divisible by 6 if and only if $t=3 r+5$. If $\alpha$ is an odd integer then Equation (2.5), becomes

$$
\gamma \equiv 4 t(\bmod 6)
$$

The integer $\gamma$ is divisible by 6 if and only if $t=3 r$. If the prime $p$ is of the form $p=3+4 t$, then we assume an integer

$$
\begin{aligned}
\gamma^{\prime} & =2^{\alpha} p-2-\varphi\left(2^{\alpha} p\right)=2^{\alpha} p-2-2^{\alpha-1} p+2^{\alpha-1} \\
& =2^{\alpha+2} t-2^{\alpha+1} t+3 \cdot 2^{\alpha}-3 \cdot 2^{\alpha-1}+2^{\alpha}-2 .
\end{aligned}
$$

Taking $\alpha$ is an even in Equation (2.6) and using relation (I), we have

$$
\gamma^{\prime} \equiv 2 t(\bmod 6) \text {. }
$$

The integer $\gamma^{\prime}$ is a multiple of 6 if and only if $t=3 r$. When $\alpha$ is an odd in Equation (2.6) then by using relation (I), we have

$$
\gamma^{\prime} \equiv 4 t+2(\bmod 6) \text {. }
$$

The integer $\gamma^{\prime}$ is a multiple of 6 if and only if $t=(3 z-1) / 2$ for some positive integer $z$.

Proposition 2.12. The integer $n=2^{\alpha} \cdot 3^{\beta}$ is an anti-totient number if and only if $\beta \in\{0,1\}$ and $0 \neq \alpha \equiv 0(\bmod 2)$.

Proof. Let $n=2^{\alpha} \cdot 3^{\beta}$ be an integer. We assume an integer

$$
k=2^{\alpha} \cdot 3^{\beta}-2-\varphi\left(2^{\alpha} \cdot 3^{\beta}\right)=2^{\alpha} \cdot 3^{\beta}-2-2^{\alpha} \cdot 3^{\beta-1} .
$$

If $\beta=0$ then by Proposition 2.10, the result holds. If $\beta=1$, then $k$ is divisible by 4 if and only if $0 \neq \alpha \equiv 0(\bmod 2)$.

Theorem 2.13. Let $p=1+4 t, q=3+4 t$ be primes for some integer $t$. Then the integer $n=2^{\alpha} p q$ is anti-totient if an only if $\alpha$ is even and $t=(3 k-1)$ or $t=(3 k+1)$ for some positive integer $k$.

Proof. Let $n=2^{\alpha} p q$ be any integer, assume an integer $\gamma$ as

$$
\begin{aligned}
\gamma & =2^{\alpha} p q-2-\varphi\left(2^{\alpha} p q\right)=2^{\alpha} p q-2-2^{\alpha-1}(p q-p-q+1) \\
& =2^{\alpha-1}(p q+p+q-1)-2 \\
& \equiv 2^{\alpha+1} t^{2}-2(\bmod 6)
\end{aligned}
$$

If $\gamma$ is an even integer then Congruence (2.7) together with relation (I), we have,

$$
\gamma \equiv 2\left(t^{2}-1\right) \equiv 2(t+1)(t-1)(\bmod 6)
$$

an integer $\gamma$ is a multiple of 6 if and only if $t+1$ or $t-1$ are multiple of three. Thus, $\gamma$ is divisible by 6 if and only if $t=3 k-1$ or $t=3 k+1$. Furthermore, we show that when $\alpha$ as an odd then $n$ is not an anti-totient number. If $\alpha$ is odd then by using the relation (I) in Congruence (2.7), we have

$$
\gamma \equiv 4 t^{2}-2(\bmod 6)
$$

Therefore, $\gamma$ is not divisible by 6 for any integer $t$. Hence, $n$ is not anti-totient.

Theorem 2.14. Let $n=2^{\alpha} \cdot \prod_{i=1}^{m} p_{i}^{\alpha_{i}}$ be an integer with each $\alpha_{i} \geq 2$. Then $n$ is an anti-totient if and only if each $p_{i} \equiv 1(\bmod 6)$ and $\alpha \equiv 1(\bmod 2)$. 
Proof. Let $n=2^{\alpha} \cdot \prod_{i=1}^{m} p_{i}^{\alpha_{i}}$ be an integer with each $\alpha_{i} \geq 2$. Note that every odd prime $p>3$ is either $p \equiv 1(\bmod 6)$ or $p \equiv 5(\bmod 6)$. We assume that,

$$
\begin{aligned}
\gamma & =2^{\alpha} \cdot \prod_{i=1}^{m} p_{i}^{\alpha_{i}}-2-\varphi\left(2^{\alpha} \cdot \prod_{i=1}^{m} p_{i}^{\alpha_{i}}\right) \\
& =2^{\alpha} \cdot \prod_{i=1}^{m} p_{i}^{\alpha_{i}}-2-2^{\alpha-1} \cdot \prod_{i=1}^{m} p_{i}^{\alpha_{i}-1}\left(p_{i}-1\right) .
\end{aligned}
$$

Next, we claim that if 6 divides $\gamma$ then there is no odd prime $p_{i}=3$ or $p_{i} \equiv 5(\bmod 6)$ in (2.9). Suppose that there is an odd prime $p_{i}=3$ in (2.9). Without any loss, assume that $p_{1}=3$. Therefore, $(2.9)$ becomes

$$
\gamma=2^{\alpha} \cdot 3^{\alpha_{1}} \cdot \prod_{i=2}^{m} p_{i}^{\alpha_{i}}-2-2^{\alpha} \cdot 3^{\alpha_{1}-1} \cdot \prod_{i=2}^{m} p_{i}^{\alpha_{i}-1}\left(p_{i}-1\right) .
$$

Since $\alpha_{i} \geq 2$. Thus, $(2.10)$ becomes $\gamma \equiv 4(\bmod 6)$. Next, assume that there is a prime of the form $p_{i} \equiv 5(\bmod 6)$ in $(2.9)$. Suppose that $p_{1} \equiv 5(\bmod 6)$, so $(2.9)$ becomes,

$$
\gamma=\left(2^{\alpha} \cdot 5^{\alpha_{1}} \cdot \prod_{i=2}^{m} p_{i}^{\alpha_{i}}-2-2^{\alpha+1} \cdot 5^{\alpha_{1}-1} \cdot \prod_{i=2}^{m} p_{i}^{\alpha_{i}-1}\left(p_{i}-1\right)\right)(\bmod 6) .
$$

For every choice of prime $p_{i}$ and $\alpha_{1}, \gamma$ is not divisible by 6 . Lastly, if $p_{i} \equiv 1(\bmod 6)$ in $(2.9)$, then we have $\gamma=2^{\alpha}-2(\bmod 6)$. Thus, $\gamma$ is multiple of 6 if and only if $\alpha \equiv 1(\bmod 2)$.

Proposition 2.15. A totient number $n$ is an anti-totient number if and only if

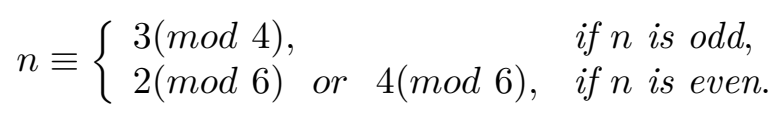

Proof. Let $n$ be a totient number then by Proposition $1.2, \varphi(n)=2^{k+1}, k \geq 1$. Since

$$
2^{k+1} \equiv \begin{cases}0(\bmod 4), & \text { for each positive ineger } k, \\ 4(\bmod 6), & \text { if } k \text { is odd, } \\ 2(\bmod 6), & \text { if } k \text { is even }\end{cases}
$$

Thus, by using Lemma 2.3 we have Proposition (2.15).

There are some integers which are deduced from binomial coefficients termed as triangular, pentagonal, and hexagonal numbers. The $n t h$ term formulas for triangular, pentagonal, and hexagonal numbers are $T_{n}=C_{2}^{n+1}=\frac{n(n+1)}{2}, P_{n}=\frac{3 n^{2}-n}{2}$, and $H_{n}=2 n^{2}-n$ respectively.

Theorem 2.16. Let $T_{2 m+1}, T_{2 n}$ be triangular numbers with $\varphi\left(T_{2 m+1}\right)=2 k, \varphi\left(T_{2 n}\right)=$ $2 r, \quad k, r>1$. We have the following characterizations,

(1) If $m \in \mathbb{E}^{+}$, then $T_{2 m+1}$ is an anti-totient number if and only if $(m, k) \in\{(a, b) \mid a \equiv$ $2(\bmod 4), b \in \mathbb{N} \backslash\{1\}\} \bigcup\{(c, d) \mid 0 \neq c \equiv 4(\bmod 4), 1 \neq d \equiv \pm 1(\bmod 4)\}$.

(2) If $m \in \mathbb{O}^{+}$, then $T_{2 m+1}$ is an anti-totient number if and only if $(m, k) \in\{(a, b) \mid a \equiv$ $\pm 1(\bmod 6), b \equiv 2(\bmod 6)$ or $b \equiv 5(\bmod 6)\} \cup\{(c, d) \mid c \equiv 3(\bmod 6), 1 \neq d \equiv$ $1(\bmod 6)$ or $d \equiv 4(\bmod 6)\}$.

(3) If $n \in \mathbb{O}^{+}$, then $T_{2 n}$ is an anti-totient number if and only if $(n, r) \in\{(a, b) \mid a \equiv$ $\left.1(\bmod 4), b \in \mathbb{E}^{+}\right\} \cup\{(c, d) \mid c \equiv 3(\bmod 4), d \equiv \pm 1(\bmod 4)\}$.

(4) If $n \in \mathbb{E}^{+}$, then $T_{2 n}$ is an anti-totient number if and only if $(n, r) \in\{(a, b) \mid a \equiv$ $2(\bmod 6), b \equiv 1(\bmod 6)$ or $b \equiv 4(\bmod 6)\} \bigcup\{(c, d) \mid c \equiv 4(\bmod 6)$, or $0 \neq c \equiv$ $6(\bmod 6), d \equiv 2(\bmod 6)$ or $d \equiv 5(\bmod 6)\}$. 
Proof. Let $T_{2 m+1}, T_{2 n}$ be triangular numbers with $\varphi\left(T_{2 m+1}\right)=2 k, \varphi\left(T_{2 n}\right)=2 r, k, r>1$. Taking $m \in \mathbb{E}^{+}$, then $T_{2 m+1}=(m+1)(2 m+1)$ is odd. So, by Lemma 2.3,

$$
2 m^{2}+3 m+2 \equiv 2 k(\bmod 4) .
$$

The Congruence (2.11) has a solution if and only if $(m, k)$ belongs to the set $\{(a, b) \mid a \equiv$ $2(\bmod 4), b \in \mathbb{N} \backslash\{1\}\} \bigcup\{(c, d) \mid 0 \neq c \equiv 4(\bmod 4), 1 \neq d \equiv \pm 1(\bmod 4)\}$. This proves $(1)$. If $m \in \mathbb{O}^{+}$, then $(m+1)(2 m+1)$ is even then by Lemma 2.3 ,

$$
2 m^{2}+3 m-1 \equiv 2 k(\bmod 6) .
$$

the Congruence (2.12) has a solution if and only if $(m, k)$ belongs to the set $\{(a, b) \mid a \equiv$ $\pm 1(\bmod 6), b \equiv 2(\bmod 6)$ or $b \equiv 5(\bmod 6)\} \cup\{(c, d) \mid c \equiv 3(\bmod 6), 1 \neq d \equiv 1(\bmod 6)$ or $d \equiv$ $4(\bmod 6)\}$.

If $n \in \mathbb{O}^{+}$, then $T_{2 n}=(n)(2 n+1)$ is an odd number. So by Lemma 2.3,

$$
2 n^{2}+n+1 \equiv 2 k(\bmod 4) .
$$

the Congruence (2.13) has a solution if and only if $(n, r)$ belongs to the set $\{(a, b) \mid a \equiv$ $\left.1(\bmod 4), b \in \mathbb{E}^{+}\right\} \bigcup\{(c, d) \mid c \equiv 3(\bmod 4), d \equiv \pm 1(\bmod 4)\}$.

If $n \in \mathbb{E}^{+}$, then $T_{2 n}=(n)(2 n+1)$ is an even number. So by Lemma 2.3,

$$
2 n^{2}+n-2 \equiv 2 k(\bmod 6) .
$$

the Congruence (2.14) has a solution if and only if $(n, r)$ belongs to the set $\{(a, b) \mid a \equiv$ $2(\bmod 6), b \equiv 1(\bmod 6)$ or $b \equiv 4(\bmod 6)\} \cup\{(c, d) \mid c \equiv 4(\bmod 6)$, or $0 \neq c \equiv 6(\bmod 6), d \equiv$ $2(\bmod 6)$ or $d \equiv 5(\bmod 6)\}$.

Theorem 2.17. Let $P_{2 n}$, and $P_{2 m+1}$ be pentagonal numbers with $\varphi\left(P_{2 m+1}\right)=2 k, \varphi\left(P_{2 n}\right)=$ $2 r, k, r>1$. We have the following characterizations.

(1) If $n \in \mathbb{E}^{+}$, then $P_{2 n}$ is an anti-totient number if and only if $(n, r) \in\{(a, b) \mid a \equiv$ $2(\bmod 6), b \equiv 1(\bmod 3)\} \bigcup\{(c, d) \mid c \equiv 4(\bmod 6), 0 \neq d \equiv 3(\bmod 3) \cup\{(e, f) \mid 0 \neq$ $e \equiv 6(\bmod 6), f \equiv 2(\bmod 3)\}$.

(2) If $n \in \mathbb{O}^{+}$, then $P_{2 n}$ is an anti-totient number if and only if $(n, r) \in\{(a, b) \mid a \equiv$ $1(\bmod 4), b \equiv 1(\bmod 2)\} \bigcup\{(c, d) \mid c \equiv 3(\bmod 4), 0 \neq d \equiv 0(\bmod 2)\}$.

(3) If $m \in \mathbb{O}^{+}$, then $P_{2 m+1}$ is an anti-totient number if and only if $(m, k) \in\{(a, b) \mid a \equiv$ $1(\bmod 6), b \equiv 2(\bmod 3)\} \bigcup\{(c, d) \mid c \equiv 3(\bmod 6), d \equiv 1(\bmod 3)$ $\bigcup\{(e, f) \mid e \equiv 5(\bmod 6), f \equiv 2(\bmod 3)\}$.

(4) If $m \in \mathbb{E}^{+}$, then $P_{2 m+1}$ is an anti-totient number if and only if $(m, k) \in\{(a, b) \mid a \equiv$ $2(\bmod 4), 0 \neq b \equiv 0(\bmod 2)\} \cup\{(c, d) \mid 0 \neq c \equiv 4(\bmod 4), d \equiv 1(\bmod 2)\}$.

The proof of Theorem 2.17 is similar to the proof of Theorem 2.16.

Theorem 2.18. Let $H_{n}$ be a hexagonal number with $\varphi\left(H_{n}\right)=2 k, k>1$. We have the following characterizations,

(1) If $n \in \mathbb{E}^{+}$, then $H_{n}$ is an anti-totient number if and only if $(n, r) \in\{(a, b) \mid a \equiv$ $2(\bmod 6), b \equiv 2(\bmod 3)\} \cup\{(c, d) \mid c \equiv 4(\bmod 6), d \equiv 1(\bmod 3)$

$\bigcup\{(e, f) \mid 0 \neq e \equiv 6(\bmod 6), f \equiv 2(\bmod 3)\}$.

(2) If $n \in \mathbb{O}^{+}$, then $H_{n}$ is an anti-totient number if and only if $(n, r) \in\{(a, b) \mid a \equiv$ $1(\bmod 4), b \equiv 1(\bmod 2)\} \bigcup\{(c, d) \mid c \equiv 3(\bmod 4), 0 \neq d \equiv 0(\bmod 2)\}$.

Proof. Let $H_{n}$ be a hexagonal number with $\varphi\left(H_{n}\right)=2 k, k>1$. If $n \in \mathbb{E}^{+}$, then $H_{n}=n(2 n-1)$ is even, so by Lemma 2.3 ,

$$
2 n^{2}-n-2 \equiv 2 k(\bmod 6) .
$$


the Congruence (2.15) has a solution if and only if $(n, r)$ belongs to the set $\{(a, b) \mid a \equiv$ $2(\bmod 6), b \equiv 2(\bmod 3)\} \bigcup\{(c, d) \mid c \equiv 4(\bmod 6), d \equiv 1(\bmod 3) \cup\{(e, f) \mid 0 \neq e \equiv 6(\bmod 6), f \equiv$ $2(\bmod 3)\}$. if $n \in \mathbb{O}^{+}$, then $H_{n}=n(2 n-1)$ is odd, so by Lemma 2.3,

$$
2 n^{2}-n+1 \equiv 2 k(\bmod 6) .
$$

The Congruence (2.16) has a solution if and only if $(n, r) \in\{(a, b) \mid a \equiv 1(\bmod 4), b \equiv$ $1(\bmod 2)\} \bigcup\{(c, d) \mid c \equiv 3(\bmod 4), 0 \neq d \equiv 0(\bmod 2)\}$.

\section{Half anti-totient numbers}

In this section, we give the notion of half anti-totient numbers and characterize them completely. Also, also establish their relations with existing well-known classes of integers.

Definition 3.1. A positive integer $n$ is called a half anti-totient number if the sum of non co-prime residues of $n$ is

$$
\sum_{r<n,(r, n) \neq 1} r= \begin{cases}n k, & \text { if } n \text { is odd and for some even } k,(k \neq 0), \\ \frac{3 n}{2} k, & \text { if } n \text { is even and for some odd } k .\end{cases}
$$

Example 3.2. The set of non coprime residues of 21 is $S=\{3,6,7,9,12,14,15,18\}$, sum of elements of $S$ is $84=21 \cdot 4$, and the set of non co-prime residues of 24 is $T=$ $\{2,3,4,6,8,9,10,12,14,15,16,18,20,21,22\}$, whose sum is $180=3 \cdot \frac{24}{2} \cdot 5$. If we take $n=15$ then the sum of its co-prime residues is 45 which can not be written in the form of $15 k$ for any even integer $k$. Therefore, 21 and 24 are odd even half anti-totient numbers respectively, but 15 is not a half anti-totient number.

Lemma 3.3. An integer $n$ is half anti-totient number if and only if

$$
\varphi(n) \equiv \begin{cases}n-1(\bmod 4), & \text { if } n \text { is odd }, \\ n+2(\bmod 6), & \text { if } n \text { is odd }\end{cases}
$$

The proof is similar to the proof of Lemma 2.3.

Proposition 3.4. Let $p, q$ be primes of the form $p=1+4 t, q=3+4 t$ for some positive integer $t$. We have the following results.

(a) The integer $p^{\alpha}$ is a half anti-totient number for each positive integer $\alpha>1$.

(b) The integer $q^{\beta}$ is a half anti-totient number if and only if $1 \neq \beta \equiv 1(\bmod 2)$.

Proof. (a) Let $p=1+4 t$ be a prime number for some positive integer $t$. We assume an integer

$$
\begin{aligned}
\gamma & =p^{\alpha}-1-\varphi\left(p^{\alpha}\right)=p^{\alpha-1}-1 \\
& \equiv(1)^{\alpha-1}-1(\bmod 4)
\end{aligned}
$$

Clearly, $\gamma$ is divisible by 4 for each positive integer $\alpha$. Therefore, by Lemma $3.3, p^{\alpha}$ is half anti-totient number.

(b) Let $q=3+4 t$ be a prime integer for some positive integer $t$. We assume an integer $\gamma^{\prime}$

$$
\begin{aligned}
\gamma^{\prime} & =q^{\beta}-1-\varphi\left(q^{\beta}\right)=q^{\beta-1}-1 \\
& \equiv(3)^{\beta-1}-1(\bmod 4)
\end{aligned}
$$

Since,

$$
3^{\beta}(\bmod 4)= \begin{cases}3, & \text { if } \beta \text { is odd, } \\ 1, & \text { if } \beta \text { is even. }\end{cases}
$$

Therefore, by using relation (II) in Congruence (3.1), $\gamma^{\prime}$ is divisible by 4 if and only if $1 \neq \beta \equiv 1(\bmod 2)$. 
Theorem 3.5. Let $n=\prod_{i=1}^{m} p_{i}^{\alpha_{i}}(m>1)$ be an integer, where $p_{i}$ are all of them odd primes. Then $n$ is a half anti-totient number if and only if one of the following holds:

(a) If all primes are of the form $p_{i} \equiv 1(\bmod 4)$.

(b) If the number of primes of the form $p_{i} \equiv 3(\bmod 4)$ is even with each $p_{i}$ has exponent either even or odd.

(c) If the number of primes of the form $p_{i} \equiv 3(\bmod 4)$ is odd and every exponent $\alpha_{i}$ is even.

Proof. Let $n=\prod_{i=1}^{m} p_{i}^{\alpha_{i}}(m>1)$ be an integer, where $p_{i}$ are all of them odd primes. We assume an integer $\gamma$ as,

$$
\gamma=\prod_{i=1}^{m} p_{i}^{\alpha_{i}}-1-\varphi\left(\prod_{i=1}^{m} p_{i}^{\alpha_{i}}\right)=\prod_{i=1}^{m} p_{i}^{\alpha_{i}}-1-\prod_{i=1}^{m} p_{i}^{\alpha_{i}-1}\left(p_{i}-1\right) .
$$

(a) If all $p_{i}$ are of the form $p_{i} \equiv 1(\bmod 4)$, then $\gamma$ is divisible by 4 . Hence by Lemma 3.3 , $n$ is a half anti-totient number.

(b) If there are even number of primes $($ say $2 k)$ of the form $p_{i} \equiv 3(\bmod 4)$ with each $\alpha_{i}$ being even then by using relation (II) in Equation (3.2), then we have

$$
\gamma \equiv \prod_{i=2 k+1}^{m} p_{i}^{\alpha_{i}}-1(\bmod 4),
$$

since remaining primes are of the form $p_{i} \equiv 1(\bmod 4)$. Thus $\gamma$, must be divisible by 4 . Hence, by Lemma $3.3 n$ is a half anti-totient number.

If there are even number of primes of the form $p_{i} \equiv 3(\bmod 4)$ with odd exponent, then by using relation (II) in Equation (3.2), we have

$$
\gamma \equiv 3^{2 k} \cdot \prod_{i=2 k+1}^{m} p_{i}^{\alpha_{i}}-1(\bmod 4)
$$

again by using relation (II) in above congruence, we have $\gamma \equiv 0(\bmod 4)$. Hence by Lemma $3.3 n$ is a half anti-totient number.

(c) If there are odd number of primes of the form $p_{i} \equiv 3(\bmod 4)($ say $2 \mathrm{k}+1)$ with each $\alpha_{i}$ being even. By using the relation (II) in Equation (3.2), we have

$$
\gamma \equiv \prod_{i=2 k+2}^{m} p_{i}^{\alpha_{i}}-1(\bmod 4)
$$

since remaining primes are of the form $p_{i} \equiv 1(\bmod 4)$. Thus, $\gamma$ is divisible by 4 .

Conversely, assume that $n$ is a half anti-totient number. On contrary, assume that $(a),(b)$ and $(c)$ do not hold. Since $n$ is half anti-totient so $\gamma$ in Equation (3.2) must be divisible by 4 . Since $(a),(b)$ and $(c)$ are not hold, so there are odd number of primes of the form $p_{i} \equiv 3(\bmod 4)$ with each prime has an odd exponent. Using relation (II) in Equation (3.2), we have

$$
\gamma \equiv 3^{2 k+1} \cdot \prod_{i=2 k+2}^{m} p_{i}^{\alpha_{i}}-1(\bmod 4),
$$

remaining primes are $p_{i} \equiv 1(\bmod 4)$ with any exponent or $p_{i} \equiv 3(\bmod 4)$ with even exponent. In both cases $\gamma \equiv 2(\bmod 4)$, which leads to a contradiction against the fact that $n$ is a half anti-totient number.

Proposition 3.6. An integer $n=2^{\alpha} \cdot 3^{\beta}$ is a half anti-totient number if and only if $(\alpha, \beta) \in\left\{(x, 0) \mid x \in \mathbb{O}^{+} \backslash\{1\}\right\} \cup\left\{(x, 1) \mid x \in \mathbb{O}^{+}\right\}$.

The proof is similar to the proof of Proposition 2.12.

In the following result we characterize the class of all even half anti-totient numbers which are not divisible by 3 . 
Theorem 3.7. Let $n=2^{\alpha} \cdot \prod_{i=1}^{m} p_{i}^{\alpha_{i}}$ be an integer so that $p_{i} \neq 3$ are odd primes greater than 3. Then $n$ is a half anti-totient number if and only if one of the following statements hold:

(a) If all primes are of the form $p_{i} \equiv 1(\bmod 6)$, with $0 \neq \alpha \equiv 0(\bmod 2)$.

(b) If there are even number of primes of the form $p_{i} \equiv 5(\bmod 6)$ with each $p_{i}$ has exponent either even or odd and $0 \neq \alpha \equiv 0(\bmod 2)$.

Proof. Let $n=2^{\alpha} \prod_{i=1}^{m} p_{i}^{\alpha_{i}}$ be an integer with $p_{i} \neq 3$ are odd primes. We assume that an integer

$$
\begin{aligned}
\gamma & =2^{\alpha} \cdot \prod_{i=1}^{m} p_{i}^{\alpha_{i}}+2-\varphi\left(2^{\alpha} \cdot \prod_{i=1}^{m} p_{i}^{\alpha_{i}}\right) \\
& =2^{\alpha} \cdot \prod_{i=1}^{m} p_{i}^{\alpha_{i}}+2-2^{\alpha-1} \cdot \prod_{i=1}^{m} p_{i}^{\alpha_{i}-1}\left(p_{i}-1\right)
\end{aligned}
$$

(a) If all primes are of the form $p_{i} \equiv 1(\bmod 6)$ then from Equation $(3.3)$, we have

$$
\gamma \equiv 2^{\alpha}+2(\bmod 6)
$$

by relation (I) an integer $\gamma$ is divisible by 6 if and only if $\alpha \equiv 0(\bmod 2)$.

Since,

$$
5^{\beta}(\bmod 6)= \begin{cases}5, & \text { if } \beta \text { is odd } \\ 1, & \text { if } \beta \text { is even. }\end{cases}
$$

(b) If there are even number of primes $($ say $2 k)$ of the form $p_{i} \equiv 5(\bmod 6)$ with each $\alpha_{i}$ being even then Equation (3.3), becomes by using relation (III)

$$
\gamma \equiv 2^{\alpha} \cdot \prod_{i=2 k+1}^{m} p_{i}^{\alpha_{i}}+2-2^{4 k+\alpha-1} \cdot 5^{2 k} \cdot \prod_{i=2 k+1}^{m} p_{i}^{\alpha_{i}-1}\left(p_{i}-1\right)(\bmod 6) .
$$

Since remaining all primes are of the form $p_{i} \equiv 1(\bmod 6)$. Hence, Congruence $(3.4)$ leads to

$$
\gamma \equiv 2^{\alpha}+2(\bmod 6)
$$

by using relation $(\mathrm{I})$, an integer $\gamma$ is a multiple of 6 if and only if $0 \neq \alpha \equiv 0(\bmod 2)$.

If there are even number of primes of the form $p_{i} \equiv 5(\bmod 6)$ with each $p_{i}$ has odd exponent then Equation (3.3), becomes

$$
\gamma \equiv 2^{\alpha} \cdot 5^{2 k} \cdot \prod_{i=2 k+1}^{m} p_{i}^{\alpha_{i}}+2-2^{4 k+\alpha-1} \cdot \prod_{i=2 k+1}^{m} p_{i}^{\alpha_{i}-1}\left(p_{i}-1\right)(\bmod 6),
$$

since remaining primes are of the form $p_{i} \equiv 1(\bmod 6)$. Thus, by using relation (III), $\gamma$ is divisible by 6 if and only if $0 \neq \alpha \equiv 0(\bmod 2)$.

Conversely, assume that $n$ is a half anti totient number. On contrary assume that conditions $(a)$ and $(b)$ do not hold. That is, there are odd number of primes $p_{i}$ (say $2 k+1$ ) of the form $p_{i} \equiv 5(\bmod 6)$ with each has exponent either even or odd. We assume that there are two integers $\gamma$ and $\gamma^{\prime}$ corresponding to each prime having exponent even or odd respectively. These integers are

$$
\gamma \equiv 2^{\alpha} \cdot \prod_{i=2 k+2}^{m} p_{i}^{\alpha_{i}}+2-2^{4 k+\alpha+1} \cdot 5^{2 k+1} \cdot \prod_{i=2 k+2}^{m} p_{i}^{\alpha_{i}-1}\left(p_{i}-1\right)(\bmod 6)
$$


and

$$
\gamma^{\prime} \equiv 2^{\alpha} \cdot 5^{2 k+1} \cdot \prod_{i=2 k+2}^{m} p_{i}^{\alpha_{i}}+2-2^{4 k+\alpha+1} \cdot \prod_{i=2 k+2}^{m} p_{i}^{\alpha_{i}-1}\left(p_{i}-1\right)(\bmod 6),
$$

clearly for any choice of $\alpha$ the integers $\gamma$ and $\gamma^{\prime}$ are not divisible by 6 , which is a contradiction against the fact that $n$ is a half anti-totient number.

Corollary 3.8. An integer $2^{\alpha}$ is a half anti-totient number if and only if $1 \neq \alpha \equiv$ $1(\bmod 2)$.

The proof is similar to the proof of Proposition 2.10.

Remark 3.9. An even integer $n$ having a factor of 9 is neither an anti-totient nor a half anti-totient number.

Proposition 3.10. A totient number $n$ is a half anti-totient number if and only if

$$
n \equiv \begin{cases}1(\bmod 4), & \text { if } n \text { is odd }, \\ 0(\bmod 6) \text { or } 2(\bmod 6), & \text { if } n \text { is even. }\end{cases}
$$

The proof is similar to the proof of Proposition 2.15.

Theorem 3.11. An even Zumkeller number $n=2^{\alpha} p_{1}^{\alpha_{1}} p_{2}^{\alpha_{2}} \cdots p_{m}^{\alpha_{m}}$ which does not have a factor of 9 is either an anti-totient or a half anti-totient number if any of the following conditions are satisfied.

(a) If $n$ is not divisible by 9.

(b) If all primes of the form $p_{i} \equiv 1(\bmod 6)$ and $0 \neq \alpha \equiv 0(\bmod 2)$.

(c) If there are even number of primes of the form $p_{i} \equiv 5(\bmod 6)$, with each $p_{i}$ has either even or odd exponent, and $0 \neq \alpha \equiv 0(\bmod 2)$.

Proof. Let $n=2^{\alpha} p_{1}^{\alpha_{1}} p_{2}^{\alpha_{2}} \cdots p_{m}^{\alpha_{m}}$ be an even Zumkeller number which has no factor of 9 . By Proposition 1.3, $n$ must has an odd prime with odd exponent. Since all odd primes can be divided into two classes namely, $p \equiv 1(\bmod 4)$ or $p \equiv 3(\bmod 4)$, also in modulo 6 these primes except 3 leave remainder 1 or 5 . That is, every odd prime except 3 in modulo 6 can be classified in the form $p \equiv 1(\bmod 5)$ or $p \equiv 5(\bmod 6)$. If $n$ has only odd prime 3 then $n=2^{\alpha} \cdot 3$, so by Proposition 2.12 and Proposition 3.6, $n$ is anti-totient and half anti-totient respectively. If all $p_{i}$ are of the form $p_{i} \equiv 1(\bmod 6)$, then we assume an integer $\gamma=n+2-\varphi(n) \Rightarrow \gamma \equiv 2^{\alpha}+2(\bmod 6)$. Thus, $\gamma$ is divisible by 6 if and only if $0 \neq \alpha \equiv 0(\bmod 2)$. Finally, if last condition meet then $n$ is a half anti-totient number by Theorem 3.7.

In Table 1, we give the comparison of anti-totient and half anti-totient numbers from first 100 positive integers.

\begin{tabular}{|c|c|}
\hline Anti-Totient Numbers & Half Anti-Totient Numbers \\
\hline $4,9,12,14,15,16$, & $6,8,21,24,25,27$, \\
$26,34,35,39,48,49$, & $28,30,32,33,45,52$, \\
$51,52,55,62,64,74$, & $57,65,66,69,70,76$, \\
$75,81,86,87,91,95$, & $77,89,93,95,96$. \\
$98,99,100$. & \\
\hline
\end{tabular}

Table 1: Comparison of anti-totient and half anti-totient numbers from first 100 positive integers.

\section{Near Zumkeller numbers and half near Zumkeller numbers}

In this section, we introduce near Zumkeller numbers and half near Zumkeller numbers to extend the idea of Zumkeller and half Zumkeller numbers which were proposed in [13]. 
Definition 4.1. Let $S=\left\{r_{i} \mid\left(r_{i}, n\right) \neq 1,0 \leq i \leq n-1\right\} \cup\{n\}$ be the set of non co-prime residues of $n$ including itself. A positive integer $n$ is said to be a near Zumkeller number if the set $S$ can be divided into two disjoint subsets with equal sums. Also, if the set $T=\left\{r_{i} \mid\left(r_{i}, n\right) \neq 1,0 \leq i \leq n-1\right\}$ can be partitioned into two disjoint subsets with equal sums then $n$ is called a half near Zumkeller number.

Example 4.2. The set non co-prime residues of 8 with itself is $S=\{2,4,6,8\}$. The subsets $A=\{2,8\}$ and $B=\{4,6\}$ of $S$ whose sums are equal. Therefore, 8 is a near Zumkeller number. Similarly the set of non co-prime residues of 25 is $T=\{5,10,15,20\}$. The set $T$ can be partitioned into two subsets as $A=\{5,20\}$ and $B=\{10,15\}$. Note that, the integer 8 is both near and half near Zumkeller. While the integer 25 is a half near Zumkeller but not near Zumkeller.

Lemma 4.3. Let $n$ be any odd integer. Then,

$$
\varphi(n) \equiv \begin{cases}n+1(\bmod 4) & \text { if and only if } n \text { is a near Zumkeller number, } \\ n-1(\bmod 4) & \text { if and only if } n \text { is a half near Zumkeller number. }\end{cases}
$$

Proof. The set of non co-prime residues of $n$ including itself is $S=\left\{a_{i} \mid\left(a_{i}, n\right) \neq 1, \quad 0 \leq\right.$ $i \leq n-1\} \cup\{n\}$, and $R=\left\{b_{i} \mid\left(b_{i}, n\right)=1, \quad 1 \leq i \leq \varphi(n)\right\}$ is the set of co-prime residues of $n$. The sum of non-coprime residues of $n$ including itself by means of the sum of residues (say $r_{i}$ ) and co-prime residues (say $r_{i}^{\prime}$ ) of $n$ we have,

$$
\sum_{i=1}^{n+1-\varphi(n)} a_{i}=\sum_{i=1}^{n+1} r_{i}-\sum_{i=1}^{\varphi(n)} r_{i}^{\prime}=\frac{n(n+1)}{2}-\frac{n \varphi(n)}{2},
$$

Since, if $\left(a_{i}, n\right) \neq 1$ then $\left(n-a_{i}, n\right) \neq 1$, so

$$
2 \cdot \sum_{i=1}^{(n+1-\varphi(n)) / 2} a_{i}=\frac{n}{2}(n+1-\varphi(n)),
$$

by hypothesis $4 \mid(n+1-\varphi(n))$, we have

$$
\Rightarrow \sum_{i=1}^{(n+1-\varphi(n)) / 2} a_{i}=\frac{n}{4}(n+1-\varphi(n)) .
$$

Conversely, assume that $n$ is near Zumkeller, but $4 \nmid(n+1-\varphi(n))$. Since $n$ is a near Zumkeller so $(n+1-\varphi(n)) / 4$ must be an integer. Thus converse is done by using the contrapositive method of proof. Similarly, for the half near Zumkeller numbers.

The following result is a simple consequence of Lemma 4.3.

Proposition 4.4. Let $p, q$, be primes. Then,

(a) The integer $q^{\alpha}$ is a near Zumkeller number if and only if $q \equiv 3(\bmod 4)$ with $0 \neq \alpha \equiv$ $0(\bmod 2)$.

(b) Every integer $p^{\beta}$, with $p \equiv 1(\bmod 4)$ is a half near Zumkeller number for each positive integer $\beta$.

(c) The integer $q^{\alpha}, \alpha \neq 0$ with $q \equiv 3(\bmod 4)$ is a half near Zumkeller number if and only if $1 \neq \alpha \equiv 1(\bmod 2)$.

(d) Every odd anti-totient number is a near Zumkeller number.

(e) Every odd half anti-totient number is a half near Zumkeller number.

The following result characterizes the set of even near Zumkeller and half near Zumkeller numbers.

Theorem 4.5. Let $n>4$ be an even integer. Then $n$ is both near Zumkeller and half near Zumkeller number if and only if $4 \mid n$. 
Proof. The proof is obvious from the Equation (4.2).

It is well-known that every even perfect number is of Euclid type. That is, it can be expressed in the form $2^{k-1}\left(2^{k}-1\right), k>1$. Therefore we have the following result.

Proposition 4.6. Every even perfect number greater than 6 is near Zumkeller and half near Zumkeller number.

There are some even numbers which are anti-totient or half anti totient numbers but not near Zumkeller or half near Zumkeller numbers respectively. Note that 8 is both near Zumkeller and half near Zumkeller, but not anti-totient. Similarly, 16 is both near Zumkeller and half near Zumkeller, but not half anti-totient number. Therefore, we have the following results which are the simple consequences of Theorem 4.5.

Proposition 4.7. Let $n=4 k, k>1$ with $\varphi(n)=2 m, m>1$ be a near Zumkeller number. Then $n$ is an anti-totient number if and only if $(k, m) \in\left\{(1+2 t, 1+t) \mid t \in Z^{+}\right\}$.

Proof. Let $n=4 k, k>1$ and $\varphi(n)=2 m, m>1$. Since, $n$ is a multiple of 4 , so $n$ is a near Zumkeller number. We assume an integer

$$
\gamma \equiv 4 k-2-2 m(\bmod 6)
$$

an integer $n=4 k$ is anti-totient if and only if $6 \mid \gamma$. Thus, $k+m \equiv 2(\bmod 3)$. By the solution linear diophantine equation, the positive solution set of $k+m \equiv 2(\bmod 3)$ is $\left\{(1+2 t, 1+t) \mid t \in \mathbb{Z}^{+}\right\}$.

Proposition 4.8. Let $n=4 k, k>1$ with $\varphi(n)=2 m, m>1$ be a half near Zumkeller number. Then $n$ is a half anti-totient number if and only if $(k, m) \in\{(2 t+2, t+2) \mid t \in$ $\left.\mathbb{Z}^{+}\right\}$.

The proof is similar to the proof of Proposition 4.7.

\section{Application of anti-totient numbers in graph labeling}

In previous sections, we have introduced the new classes of integers by using the notion of non co-prime residues of a positive integer $n$. It would be more interesting and of great worth if these numbers could be employed in some well known mathematics. In our previous work, the labeling of some well known classes of graphs by means of super totient numbers have been introduced in $[11,12]$.

In this section, we propose the labeling of graphs using the notion of anti-totient numbers. The rest of the newly defined labeling over other classes can be validated in a similar technique.

Definition 5.1. [8] A full K-ary tree (sometimes k-tree) is a tree in which every vertex other than leaves has $k$ children. A full $\mathrm{K}$-ary tree is a tree where each internal vertex has degree $k+1$, each leaf has degree 1 and the root vertex has degree $k$. If $\mathrm{k}$ is an odd then every full K-ary tree has even number of vertices and if $k$ is even, then every full K-ary tree has odd number of vertices. If a full K-ary tree has $m$ leaves then it has $\sum_{i=0}^{m} k^{i}$ vertices and $\sum_{i=0}^{m} k^{i}-1$ edges.

Definition 5.2. Let $G=(V, E)$ be a graph. A mapping $\xi: V \rightarrow \mathbb{N}$ admits an anti-totient labeling if there exists an induced function $\xi^{*}: E \rightarrow \mathbb{N}$ defined by $\xi^{*}(x y)=\xi(x) \xi(y)$ and assigns an anti-totient number to each edge of $G$.

Definition 5.3. A graph $G$ is said to be an anti-totient graph if $G$ admits an anti-totient labeling. 
Example 5.4. An anti-totient graph for vertex set $\{2,4,5,6,7,8\}$ is shown in Fig.1.

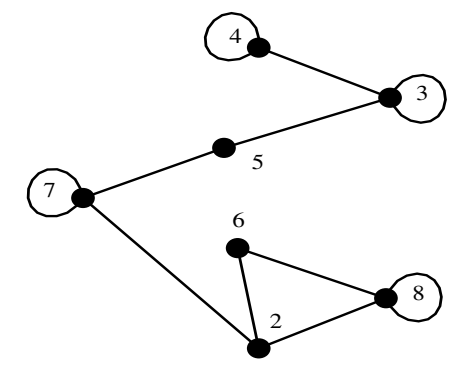

Fig.1: Anti-Totient Graph

Theorem 5.5. A full K-ary tree is an anti-totient graph.

Proof. Let $V=\left\{a_{00}, a_{i j} \mid 1 \leq i \leq m, 1 \leq j \leq k^{m}\right\}$ and $E=\left\{a_{00} a_{1 j} \mid 1 \leq j \leq k\right\} \cup$ $\left\{a_{r j} a_{r+1, j} \mid 1 \leq r \leq m-1,1 \leq i \leq k^{r}, \quad k i-1 \leq j \leq k i\right\}$ be the edge set of full binary tree T. The injective function $\xi: V \rightarrow \mathbb{N}$ is defined as follows:

$$
\xi\left(a_{i j}\right)= \begin{cases}1, & \text { if } i, j=0, \\ 2^{2 i}, & \text { if } i=1,1 \leq j \leq k, \\ 7^{i}, & \text { if } i=2,1 \leq j \leq k^{2}, \\ 2^{2 j+2 k\left(\sum_{l=0}^{\frac{i-3}{2}} k^{2 l}\right)}, & \text { if } i \geq 3 \text { and, } i \equiv 1(\bmod 2), 1 \leq j \leq k^{i}, \\ 7^{j+k^{2}\left(\sum_{l=0}^{\frac{i-4}{2}} k^{2 l}\right)}, & \text { if } i \geq 4 \text { and, } i \equiv 0(\bmod 2), 1 \leq j \leq k^{i} .\end{cases}
$$

The induced mapping $\xi^{*}$ is defined by

$$
\xi^{*}\left(a_{00} a_{1 i}\right)=\xi\left(a_{00}\right) \xi\left(a_{1 j}\right), \quad 1 \leq j \leq k,
$$

$\xi^{*}\left(a_{r i} a_{r+1 j}\right)=\xi\left(a_{r i}\right) \xi\left(a_{r+1 j}\right)$ where, $1 \leq r \leq m-1$, and $1 \leq i \leq k^{r}, k i-1 \leq j \leq k i$.

By using the definition of $\xi\left(a_{i j}\right)$, we have

$$
\begin{aligned}
\xi^{*}\left(a_{00} a_{1 i}\right) & =\xi\left(a_{00}\right) \xi\left(a_{1 i}\right)=2^{2 i}, \quad 1 \leq i \leq k, \\
\xi^{*}\left(a_{1 i} a_{2 j}\right) & =\xi\left(a_{1 i}\right) \xi\left(a_{2 j}\right)=2^{2 i} \cdot 7^{j}, \quad 1 \leq i \leq k, \quad 1 \leq j \leq k^{2}, \\
\xi^{*}\left(a_{2 i} a_{3 j}\right) & =\xi\left(a_{2 i}\right) \xi\left(a_{3 j}\right) \\
& =7^{i} \cdot 2^{2 j+2 k\left(\sum_{l=0}^{\frac{i-3}{2}} k^{2 l}\right)}, \quad 1 \leq i \leq k^{2}, \quad 1 \leq j \leq k^{3} .
\end{aligned}
$$

If $r \equiv 1(\bmod 2), 3 \leq r \leq m-1$ and $1 \leq i \leq k^{r}, k i-1 \leq j \leq k i$. Then,

$$
\begin{aligned}
\xi^{*}\left(a_{r i} a_{r+1 j}\right) & =\xi\left(a_{r i}\right) \xi\left(a_{r+1 j}\right) \\
& =2^{2 i+2 k\left(\sum_{l=0}^{\frac{r-3}{2}} k^{2 l}\right)} \cdot 7^{i+k^{2}\left(\sum_{l=0}^{\frac{r-4}{2}} k^{2 l}\right)} .
\end{aligned}
$$

If $r \equiv 0(\bmod 2), 4 \leq r \leq m-1$ and $1 \leq i \leq k^{r}, k i-1 \leq j \leq k i$. Then,

$$
\begin{aligned}
\xi^{*}\left(a_{m i} a_{m+1 j}\right) & =\xi\left(a_{m i}\right) \xi\left(a_{m+1 j}\right) \\
& =7^{i+k^{2}\left(\sum_{l=0}^{\frac{r-4}{2}} k^{2 l}\right)} \cdot 2^{2 i+2 k\left(\sum_{l=0}^{\frac{r-3}{2}} k^{2 l}\right)} .
\end{aligned}
$$

From Lemma 2.3, equations (5.1)-(5.5) admit an anti-totient number.

Corollary 5.6. Every full binary tree admits an anti-totient labeling. 
Example 5.7. The 3-ary tree with 3 level anti-totient graph as shown in Fig.2.

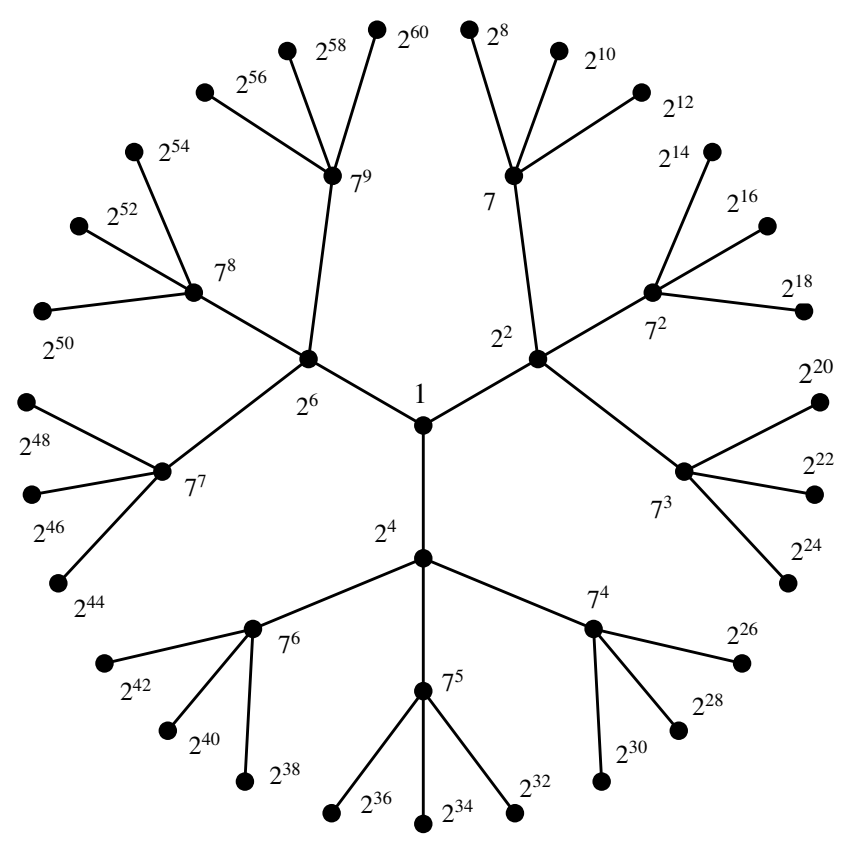

Fig.2: Anti-Totient 3-ary Tree with 3 Level.

\section{Algorithm For Anti-Totient Labeling of Full K-ary Tree}

This algorithm computes the integers to the vertices of the full $\mathrm{K}$-ary tree to label the edges with anti-totient numbers.

1: A full K-ary tree $\mathrm{T}$ with $m$ levels having $\sum_{i=0}^{m} k^{i}$ vertices and $\sum_{i=0}^{m} k^{i}-1$ edges. $V=$ $\left\{a_{00}, a_{i j} \mid 1 \leq i \leq m, 1 \leq j \leq k^{m}\right\}$ vertex set of $T$ and $E=\left\{a_{00} a_{1 j} \mid 1 \leq j \leq k\right\} \cup$ $\left\{a_{r j} a_{r+1, j} \mid 1 \leq r \leq m-1,1 \leq i \leq k^{r}, \quad k i-1 \leq j \leq k i\right\}$

$\xi\left(a_{00}\right)=1 / / \xi$ is a function defined on the vertex set of $\mathrm{T}$.

2: for $i:=1$ to $k$ do

$\xi\left(a_{1 i}\right)=2^{2 i}, \xi^{*}\left(a_{00} a_{1 j}\right)=\xi\left(a_{00}\right) \xi\left(a_{1 j}\right) / / \xi^{*}$ is an induced function.

for $j:=1$ to $k^{2}$ do

$\xi\left(a_{2 j}\right)=7^{j}$

for $i:=1$ to $k$ do,

for $j:=k i-1$ to $k i$ do

$\xi^{*}\left(a_{1 i} a_{2 j}\right)=\xi\left(a_{1 i}\right) \xi\left(a_{2 j}\right)$,

for $r:=3$ to $m$ do,

for $i:=1$ to $k^{r}$ do

if $r \equiv 1(\bmod 2)$ then,$\quad \xi\left(a_{r i}\right)=2^{2 i+2 k\left(\sum_{l=0}^{\frac{r-3}{2}} k^{2 l}\right)}$.

else

$\xi\left(a_{r i}\right)=7^{i+k^{2}\left(\sum_{l=0}^{\frac{r-4}{2}} k^{2 l}\right)}$

for $r:=2$ to $m-1$ do,

for $i:=1$ to $k^{r}$ do ,

for $j:=k i-1$ to $k i$ do

$\xi^{*}\left(a_{r i} a_{r+1 j}\right)=\xi\left(a_{r i}\right) \xi\left(a_{r+1 j}\right)$,

$\operatorname{return}(\mathbf{T})$;

3: Anti-totient k-ary tree. 


\section{Conclusion}

In this paper, we investigated four new classes of integers depending on non-relatively prime residue and their sums, named as anti-totient, half anti-totient, near Zumkeller, and half near Zumkeller. We characterized these classes completely and investigated their relationship with existing well-known classes of integers such as perfect, totient, triangular, pentagonal, and hexagonal numbers. The applications of defined classes have been proposed and validated via graph labeling. An algorithm for anti-totient labeling over $K$ ary tree has also been proposed to establish more algorithms for other classes. In future, one can work on restricted anti-totient graphs by restricting the vertex set of a graph $G$ to $\{1,2,3, \cdots, n\}$. The edge set could be defined as, there will be an edge between two integers $m$ and $n$ if their product is an anti-totient number. Furthermore, one can also define some well-known terms of graph theory on restricted anti-totient graphs such as index, metric dimension, and spectral etc.

Acknowledgment. The authors want to express their gratitude to the anonymous referees for the comprehensive reading of the paper and their pertinent comments and suggestions, which helped improve the manuscript.

\section{References}

[1] S. Babitha and J. Baskar, Prime cordial labeling on graphs, Int. J. Math. Math. Sci. 7 (1), 43-48, 2013.

[2] B.J. Balamurugan, K. Thirusangu and D.G. Thomas, Algorithms for Zumkeller labeling of full binary trees and square grids, Artif. Intell. Evol. Algorithms Eng. Syst. Springer India, 183-192, 2015.

[3] D.M. Burton, Elementary Number Theory, McGraw-Hill, 2007.

[4] S. Clark, J. Dalzell, J. Holliday, D. Leach, M. Liatti and M. Walsh, Zumkeller numbers, In Math. Abund. Conf. (Illinois State Univ.), April 18th, 2008.

[5] V.H. Dinh and M. Rosenfeld, A new labeling of $C_{2 n}$ proves that $K_{4}+M_{6 n}$ decomposes $K_{6 n}+4$, Ars Combin. 4, 255-267, 2018.

[6] K. Eshghi and P. Azimi, Applications of mathematical programming in graceful labeling of graphs, J. Appl. Math. 1, 1-8, 2004.

[7] J.A. Gallian, A dynamic survey of graph labeling, Electron. J. Comb. 16 (6), 1-219, 2009.

[8] F. Harary, Graph Theory, Addision-Wesley, 1972.

[9] J. Harrington and T.W. Wong, On super totient numbers and super totient labelings of graphs, Discrete Math. 343 (2), 111670, 2020.

[10] M. Hussain and M. Tabraiz, Super d-anti-magic labeling of subdivided $k C_{5}$, Turkish J. Math. 39 (5), 773-783, 2015.

[11] M. Khalid and A. Shahbaz, A Novel Labeling Algorithm on Several Classes of Graphs, Punjab Univ. J. Math. 49, 23-35, 2017.

[12] M. Khalid and A. Shahbaz, On Super Totient Numbers, With Applications And Algorithms To Graph Labeling, Ars Combin. 2, 29-37, 2019.

[13] Y. Peng and K.R. Bhaskara, On Zumkeller Numbers, J. Number Theory, 133 (4), 1135-1155, 2013.

[14] A. Rosa, On certain valuations of the vertices of a graph, Theory of Graphs (Internat. Symposium, Rome, July 1966), Gordon and Breach, N. Y. and Dunod Paris, 349-355, 1967.

[15] A. Shahbaz and M. Khalid, New Numbers on Euler's Totient Function with Applications, J. Math. Ext. 14 (1), 61-83, 2020.

[16] M. Seoud and S. Salman, Some results and examples on difference cordial graphs, Turkish J. Math. 40 (2), 417-427, 2016. 
[17] M. Seoud and M. Salim, Further results on edge-odd graceful graphs, Turkish J. Math. 40 (3), 647-656, 2016.

[18] S. Somasundaram and R. Ponraj, Mean labelings of graphs, Natl. Acad. Sci. Lett. 26 (7), 210-213, 2003. 